\title{
The Real Solar Neighborhood Protostars
}

\author{
Amanda L. Heiderman ${ }^{1,2,3}$ and Neal J. Evans II $^{4}$ \\ ${ }^{1}$ Department of Astronomy, University of Virginia, \\ P.O. Box 400325, Charlottesville, VA 22904, USA \\ email: heiderman@virginia.edu \\ ${ }^{2}$ National Radio Astronomy Observatory, \\ 520 Edgemont Road, \\ Charlottesville, VA 22903, USA \\ ${ }^{3}$ NSF Astronomy and Astrophysics Postdoctoral Fellow \\ ${ }^{4}$ Department of Astronomy, The University of Texas at Austin, \\ 2515 Speedway, Stop C1400, \\ Austin, Texas 78712-1205, USA \\ email: nje@astro.as.utexas.edu
}

\begin{abstract}
Characterization of how dense molecular cores evolve into stars has historically been made through observational changes in their 2 to $25 \mu \mathrm{m}$ spectral energy distribution (SED) or bolometric temperature via the Class system. Linking these observational classes to a physical protostellar phase or Stages in a consistent manner remains challenging. In order to provide a uniform indicator of whether an observationally classified embedded protostar candidate is likely to be a physical phase Stage 0 or I protostar, we performed an $\operatorname{HCO}^{+}(J=3-2)$ survey of Class $0+\mathrm{I}$ and Flat SED young stellar objects (YSOs) in the Spitzer nearby (D $<500 \mathrm{pc}$ ) Gould Belt cloud surveys. We use criteria from van Kempen et al.(2009) to classify sources as Stage $0+\mathrm{I}$ or bona fide protostars and find $84 \%$ of our $\mathrm{HCO}^{+}$detected sources meet that criteria. We recommend 0.54 Myr as an evolutionary timescale for these embedded protostars. We discuss trends in our sample with spatial distribution, molecular cloud extinction, spectral index, and bolometric temperature and luminosity.
\end{abstract}

Keywords. stars: formation, stars: fundamental parameters (classification, colors, luminosities, masses, radii, temperatures, etc.), ISM: molecules, Galaxy: solar neighborhood,ISM: clouds, ISM: dust, extinction

\section{Summary of Results}

Linking these observational classes to a physical protostellar stage in a consistent manner remains challenging. We identify Stage 0+I protostars (i.e., those with a substantial dense gas envelope) among the sample of Class $0+\mathrm{I}$ and Flat SED YSOs in the Spitzer nearby $(\mathrm{D}<500$ pc) Goulds Belt Surveys Dunham et al.(2015).

Using single pointings of $\mathrm{HCO}^{+}(J=3-2)$ at source positions and the threshold integrated intensity $\left(\mathrm{I}_{M B}\right) \mathrm{HCO}^{+}$emission criterion from van Kempen et al.(2009) to define a Stage 0+I protostar $\left(\mathrm{I}_{M B} \geqslant 0.68 \mathrm{~K} \mathrm{Km} \mathrm{s}^{-1}\right)$, in Heiderman \& Evans (2015) we find:

- $72 \%$ of Class $0+\mathrm{I}$ and $48 \%$ of Flat SED sources $\left(84 \% \mathrm{HCO}^{+}\right.$detected) are physical Stage $0+\mathrm{I}$ protostars.

- The concentration of the youngest YSOs to regions of high $A_{V}$ (i.e., Heiderman et al.(2010)) is strengthened when considering Stage $0+\mathrm{I}$ protostars ( $89 \%$ lie at $A_{V}>8$ mag; Fig. 1).

- The contamination fraction defined as the number of undetected Class 0+I plus Flat SED sources divided by the total surveyed is $>90 \%$ at $A_{V}<4$ mag and decreases with increasing $A_{V}$ (Fig. 1).

- $\mathrm{HCO}^{+}$emission strength versus evolutionary indicators $(\alpha$-the spectral index between 2$24 \mu \mathrm{m}, T_{b o l}$, and $\left.L_{b o l}\right)$ : testing the data for a $3 \sigma$ correlation, we require the absolute values of the Pearson correlation coefficient to exceed $3 /(\mathrm{N}-1)^{0.5}$ or $|r|>0.16$. We find trends for stronger $\mathrm{I}_{M B}$ with larger $\alpha$, smaller $T_{b o l}$, and no significant correlation for $L_{b o l}$. 

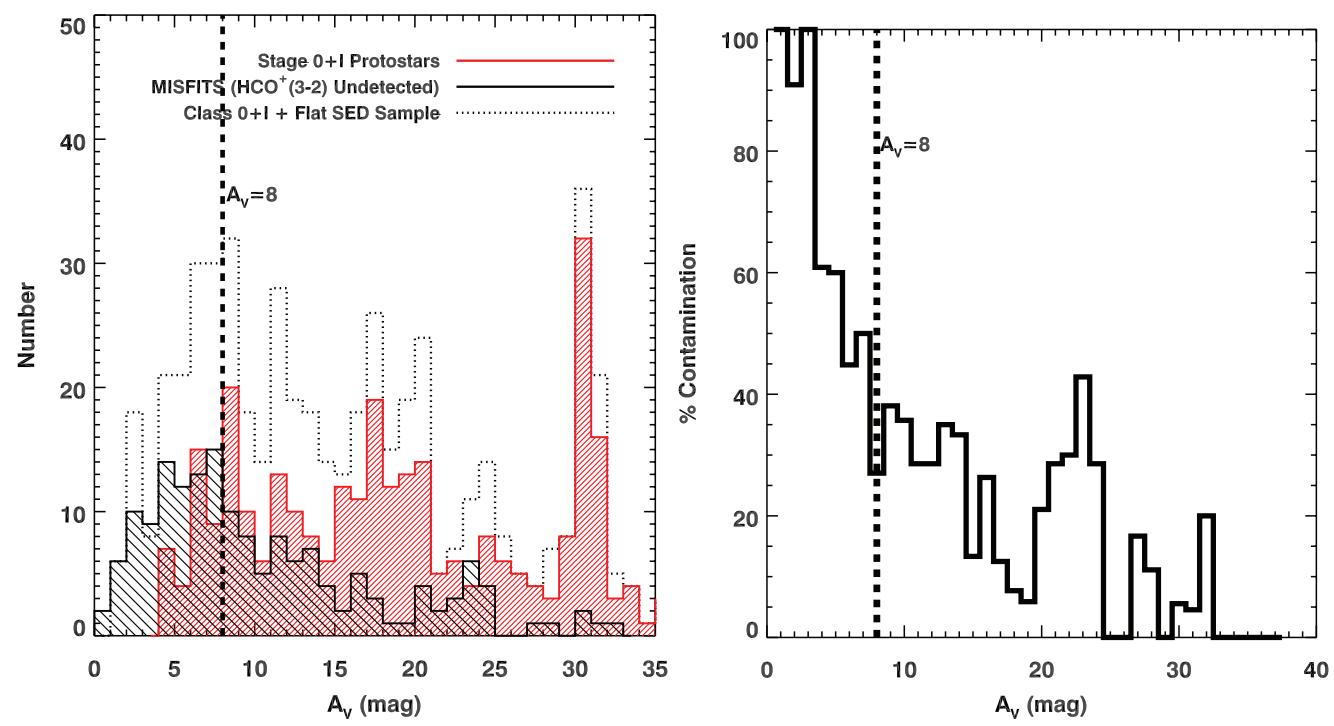

Figure 1. Left: The concentration of the youngest YSOs to regions of high $A_{V}$ (i.e., Heiderman et al.(2010)) is strengthened when considering Stage $0+\mathrm{I}$ protostars $\left(89 \%\right.$ lie at $A_{V}>8$ mag.) Right: The contamination fraction defined as the number of undetected Class $0+\mathrm{I}$ plus Flat SED sources divided by the total surveyed is $>90 \%$ at $A_{V}<4$ mag and decreases with increasing $A_{V}$

- The fraction of Stage 0+I protostars shows a strong, smooth correlation with evolutionary indicators $\left(\alpha, \log T_{b o l}\right.$, and $\left.\log L_{b o l}\right)$.

- The Stage 0+I fraction increases strongly with $\alpha$ through the Flat SED class and is $80 \%$ or greater for sources with $\alpha>0.75$.

- There is a good, but imperfect agreement with far-IR or submillimeter detections among the Stage $0+\mathrm{I}$ protostars sampled.

- The most appropriate timescale for the entire embedded Stage 0+I protostellar phase is $0.54 \mathrm{Myr}$.

Acknowledgements:The authors acknowledge funding from NSF AAPF award AST-1302978 and NSF Grant AST-1109116.

\section{References}

Heiderman, A.L., \& Evans, N.J., II 2015, ApJ, 806, 231

Dunham, M.M., Allen, L.E.; Evans, N.J., II, Broekhoven-Fiene, H., Cieza, L.A., Di Francesco, J., Gutermuth, R.A., Harvey, P.M., Hatchell, J. \& Heiderman, A. L. 2015, ApJS, 220, 11

Heiderman, A.L., Evans, N.J., II, Allen L.E., Huard, T., Heyer, M. 2010,ApJ, 723,197

van Kempen, T. A., van Dishoeck, E.F., Salter, D.M., Hogerheijde, M.R., Jorgensen, J.K., Boogert, A.C.A. $2009, A A, 498,167$ 